\title{
Electrochemical Potentials (Quasi-Fermi Levels) and the Operation of Hot-Carrier, Impact-Ionization, and Intermediate-Band Solar Cells
}

\author{
A. Martí \\ and A. Luque
}

\begin{abstract}
In the framework of the so-called third generation solar cells, three main concepts have been proposed in order to exceed the limiting efficiency of single-gap solar cells: the hot-carrier solar cell, the impact-ionization or multiple-exciton-generation solar cell, and the intermediate-band solar cell. At first sight, the three concepts are different, but in this paper, we illustrate how all these concepts, including the single-gap solar cell, share a common trunk that we call "core photovoltaic material." We demonstrate that each one of these next-generation concepts differentiates in fact from this trunk depending on the hypotheses that are made about the physical principles governing the electron electrochemical potentials. In the process, we also clarify the differences between electron, phonon, and photon chemical potentials (the three fundamental particles involved in the operation of the solar cell). The in-depth discussion of the physics involved about the operation of these cells also provides new insights about the operation of these cells.
\end{abstract}

Index Terms-Hot-carrier, impact-ionization, intermediate band, solar cells, thermodynamics.

\section{INTRODUCTION}

$\mathbf{H}$ OT-CARRIER [1], intermediate-band [2], impactionization [3], and multiple-exciton-generation solar cells [4] are novel solar cells proposed to exceed the efficiency limit of single-gap solar cells [5]. Their fundamental models have been reviewed several times and, each time, our understanding of their operation improves or their practical implementation becomes more feasible [6]-[12]. However, a unified treatment of all these cells at a fundamental level is lacking, and this paper is intended to fill this gap. Electrochemical potentials (or quasiFermi levels) will serve as the basis for this unified treatment.

A single-gap solar cell [see Fig. 1(a)] absorbs photons with energy higher than the semiconductor bandgap $E_{G}$. Its efficiency is limited, on one hand, by the fact that photons with energy lower than the bandgap are not absorbed. On the other hand, photogenerated electrons quickly relax to the bottom of the bands by giving away their energy to phonons. The output voltage of the single-gap solar cell $V$, equals the electron and hole quasi-Fermi level split $\left(\mu_{e, C}-\mu_{e, V}\right)$ divided by the

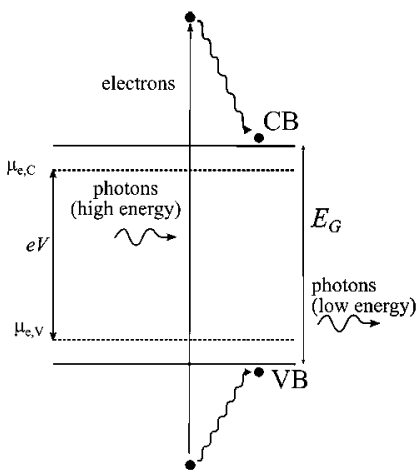

(a)

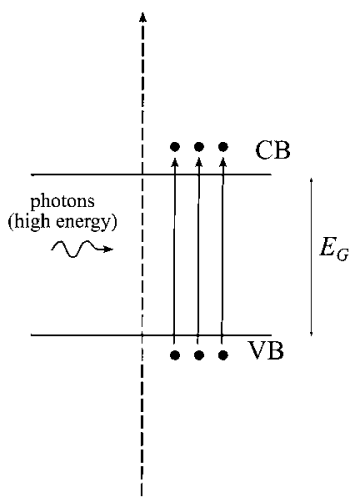

(c)

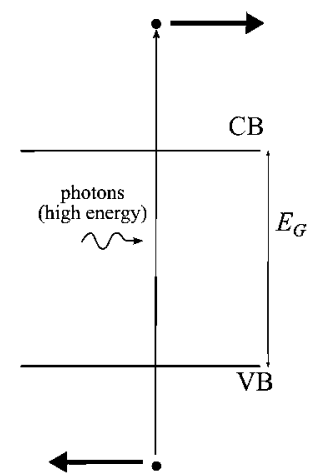

(b)

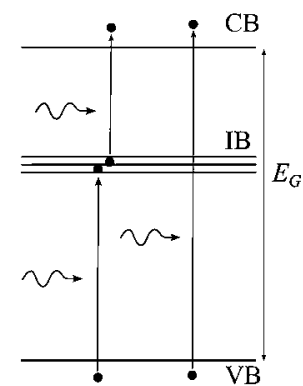

(d)
Fig. 1. Mechanism involved in the operation of the (a) single-gap solar cell, (b) HCSC, (c) IISC/MEGSC, and (d) IBSC.

electron charge. The cell emits photons whose chemical potential also equals this split. Throughout this paper, $\mathrm{eV}$ will be the electron charge multiplied by the output voltage of the cell.

To fight the energy losses of the single-gap solar cell, the hotcarrier solar cell (HCSC) proposes that photogenerated carriers are extracted from the cell before they recombine [see Fig. 1(b)]. This leads to a model in which, contrary to the intuition gained from the operation of single-gap solar cells, there is no electron and hole quasi-Fermi level split in the material that absorbs the light at the time the cell is still capable of producing output voltage.

Aiming also to optimize the use of the energy provided by the absorption of high-energy photons, the impact-ionization solar cell (IISC), and also the so-called multiple-excitation-generation solar cell (MEGSC), pursues that the absorption of a high-energy photon creates more than one electron-hole pair [see Fig. 1(c)]. 
As in the single-gap solar cell, the output voltage of this cell, multiplied by the electron charge, equals the electron and hole quasi-Fermi level split. However, the chemical potential of the emitted photons is not equal to this split but $M$ times, $M$ being the number of electron-hole pairs generated.

The intermediate-band solar cell (IBSC), on the other hand, aims to exploit the energy of the below-bandgap energy photons that are not absorbed in a single-gap solar cell. For that, it postulates a material defined by the existence of an electronic band (the intermediate band, IB) additional to the conduction band (CB) and valence band (VB). As illustrated in Fig. 1(c), the IB allows the absorption of below-bandgap energy photons through electronic transitions from the VB to the IB and from the IB to the CB. In this cell, associated with each of the bands, three quasi-Fermi levels exist instead of two.

In spite of their apparently different operation, we shall show that these cells share a common trunk that we call "core photovoltaic material" (described in Section II). In subsequent sections, we will discuss how the novel solar cells sprout from this trunk as branches depending on the hypotheses made. For that, we will assume local equilibrium thermodynamics valid so that the description of the systems by thermodynamic variables such as temperature and electrochemical potentials still makes sense even in nonequilibrium [13]-[15]. The process will also lead us to better understand why the relationships between electron and hole quasi-Fermi levels, cell output voltage, and emitted photons electrochemical potentials are apparently so different from one cell to another.

\section{Core Photovoltaic Material}

The core photovoltaic material is described by the set of energy states available to the electrons. It will be useful to think of this material as being semiconductor-like so that one set of these energy states is grouped into a VB and another set, with higher energy, is grouped into a CB. The two bands are separated by the bandgap $E_{G}$. In this core material, we will assume that electrons can only interact among themselves. This interaction is symbolically represented by the following reaction:

$$
e_{1}+e_{2} \leftrightarrow e_{3}+e_{4}
$$

where $e_{i}$ means an electron at an electronic state with energy $\varepsilon_{i}$. We first address the most general case and assume that the energy levels in (1) can be located anywhere; for example, $e_{1}$ can be in the $\mathrm{CB}$ and $e_{2}$ in the $\mathrm{VB}$, or both can be in the $\mathrm{CB}$ or both in the VB. In this way, (1) is suitable for describing all the processes illustrated in Fig. 2. We refer to the processes illustrated in Fig. 2(a) and (b) as intraband scattering processes. In these intraband processes, all the interacting electrons remain in the same band after the interaction. Processes such as the ones illustrated in Fig. 2(c)-(e) are referred to as interband scattering processes. They are characterized by their involvement with electrons in both the $\mathrm{CB}$ and the VB. In particular, the process illustrated in Fig. 2(c) would correspond to Auger recombination, and the process illustrated in Fig. 2(d) would correspond to an Auger generation or impact-ionization process. The process illustrated in Fig. 2(d) is referred to as Auger thermalization.

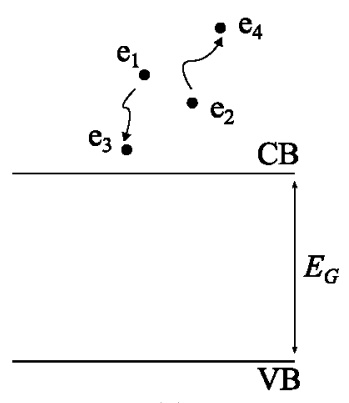

(a)

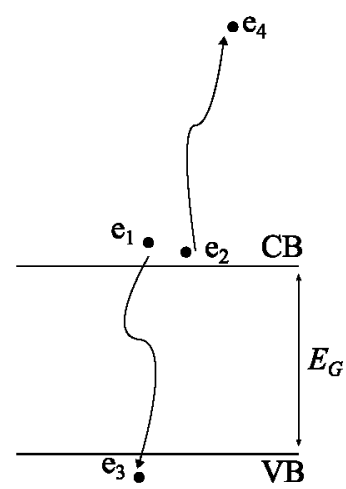

(c)

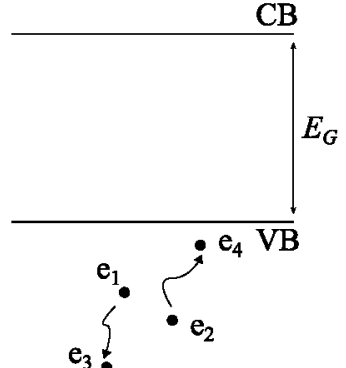

(b)

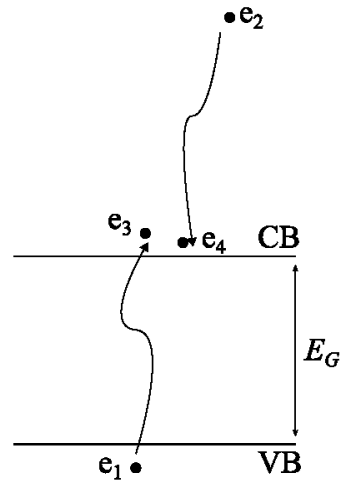

(d)

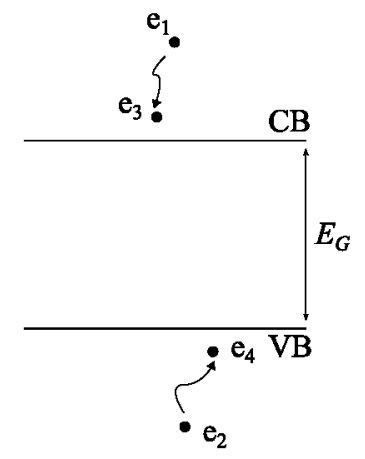

(e)

Fig. 2. Illustration of different electron-electron interactions. Two electrons interacting within (a) the CB and (b) the VB. (c) Auger recombination and (d) generation (also called impact ionization). (e) Auger thermalization.

Assuming the electron interactions from (1), we can write:

$$
\mu_{1}+\mu_{2}=\mu_{3}+\mu_{4}
$$

where $\mu_{i}$ represents the electrochemical potential or quasiFermi level of an electron at energy state $\varepsilon_{i}$ that can be degenerated without loss of generality in our argumentation. Energy conservation implies that

$$
\varepsilon_{1}+\varepsilon_{2}=\varepsilon_{3}+\varepsilon_{4} .
$$

Hence, because (2) must be fulfilled for any set of energies satisfying (3) [6], [15], $\mu_{i}$ must be a linear function of $\varepsilon_{i}$

$$
\mu_{i}=a \varepsilon_{i}+b
$$

where $a$ and $b$ are constants. This implies that if electrons in the semiconductor are at temperature $T_{c}$ (cold electron temperature), the probability $f_{\mathrm{FD}}$ of whether they occupy the state $\varepsilon_{i}$ 
follows the Fermi-Dirac distribution given by

$$
f_{\mathrm{FD}}=\frac{1}{\exp \left(\frac{\varepsilon_{i}-a \varepsilon_{i}-b}{k T_{c}}\right)+1}=\frac{1}{\exp \left(\frac{\varepsilon_{i}-\mu_{e}}{k T_{h}}\right)+1}
$$

where

$$
\begin{aligned}
T_{h} & =T_{c} /(1-a) \\
\mu_{e} & =b /(1-a) .
\end{aligned}
$$

These equations where first deduced by Würfel [6] and imply that our electron distribution can be visualized, instead of as a distribution at temperature $T_{c}$ and an energy-dependent electrochemical potential, as a distribution at an equivalent (hot) temperature $T_{h}$ and an energy-independent electrochemical potential $\mu_{e}$. Notice that, under this constant-hot-electrontemperature description, the difference in the electrochemical potential between electrons in the CB and VB would be zero because $\mu_{e}$ is the same for electrons in both bands. The basis of this conclusion is the fact that electrons in the CB have been allowed to interact with electrons in the VB. This interaction can consist of not only Auger generation recombination [see Fig. 2(c) and (d)] but also Auger thermalization [see Fig. 2(e)].

To make a solar cell, our core material must interact with photons. This interaction takes place through radiative generationrecombination processes and implies that a photon can be absorbed by pumping an electron from the VB to the $\mathrm{CB}$ (generation) and also that an electron can recombine with a hole by emitting a photon (luminescent photon). Because this photon can be reabsorbed again, luminescent photons and electronhole pairs stay in equilibrium through the reaction

$$
e_{i, \mathrm{VB}}+h v \leftrightarrow e_{i, \mathrm{CB}}
$$

where $h v$ represents a photon and $e_{i, \mathrm{VB}}$ and $e_{i, \mathrm{CB}}$ an electron in the $\mathrm{VB}$ and $\mathrm{CB}$, respectively. This equation leads us to visualize the photons in the core material as a gas at the hot-electron temperature $T_{h}$ and chemical potential $\mu_{h v}$

$$
\mu_{h v}=\mu_{e}-\mu_{e}=0
$$

because according to (7), $\mu_{e}$ is the same for electrons in the VB and the $\mathrm{CB}$.

To this point, we have not discussed electron interaction with phonons. This interaction is introduced in the later stages of this study as our description of the different solar cell models progresses. However, in advance of that discussion, notice at this stage that if phonons are assumed to have zero chemical potential, $\mu_{\text {phonon }}=0$, we can write the following reaction representing electron-phonon interaction:

$$
e_{i}+\text { phonon } \leftrightarrow e_{j}
$$

where, given the low value of the phonon energy, $e_{i}$ and $e_{j}$ both represent, for example, electrons in the $\mathrm{CB}$. We arrive at

$$
a_{C} \varepsilon_{i}+b_{C}+\mu_{\text {phonon }}=a_{C} \varepsilon_{j}+b_{C} \Rightarrow a_{C}=0
$$

which implies [based on (6)] that electrons are not hot anymore but at the temperature $T_{c}$, which can now be interpreted as the "lattice temperature" or room temperature. Similar equations would hold for electrons in the VB and leading to $a_{V}=0$. For

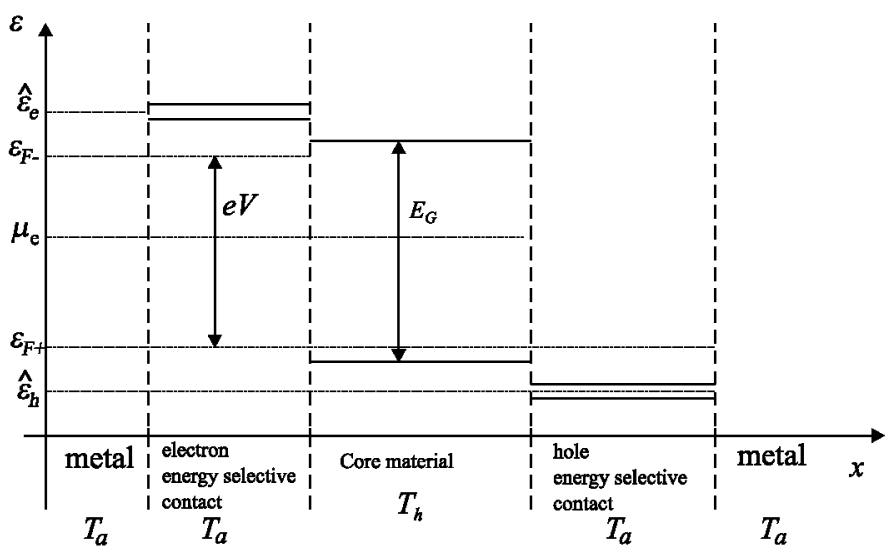

Fig. 3. Illustration of energy-selective contacts in the HCSC.

the moment, electron-phonon interactions are assumed to not occur.

\section{Hot-CARrier SOLAR CELl}

Having a material capable only of interacting with photons is not sufficient to make an actual solar cell. Selective contacts allowing only particles with some prescribed characteristic to pass through must be placed on this material. One type of conceivable, but perhaps unusual, contact is the "energy-selective" contact. Its use was proposed by Würfel [6] to preserve the physical consistency of the HCSC model. As illustrated in Fig. 3, the energy-selective contacts allow only electrons with energy $\hat{\varepsilon}_{e}$ in the $\mathrm{CB}$ and holes with energy $\hat{\varepsilon}_{h}$ to exit the core material toward the metallic contacts. The purpose of these contacts is to reversibly cool the electrons from their hot temperature $T_{h}$ to the room temperature of the contacts $T_{a}$ by increasing their electrochemical potential. In this way, electrons and holes in the metal acquire an electrochemical potential, $\varepsilon_{F}^{+}$and $\varepsilon_{F}^{-}$, respectively, given by

$$
\begin{aligned}
& \frac{\hat{\varepsilon}_{e}-\mu_{e}}{k T_{h}}=\frac{\hat{\varepsilon}_{e}-\varepsilon_{F}^{+}}{k T_{a}} \Rightarrow \varepsilon_{F}^{+}=\left(1-\frac{T_{a}}{T_{h}}\right) \hat{\varepsilon}_{e}-\mu_{e} \frac{T_{a}}{T_{h}} \\
& \frac{\hat{\varepsilon}_{h}-\mu_{e}}{k T_{h}}=\frac{\hat{\varepsilon}_{h}-\varepsilon_{F}^{-}}{k T_{a}} \Rightarrow \varepsilon_{F}^{-}=\left(1-\frac{T_{a}}{T_{h}}\right) \hat{\varepsilon}_{h}-\mu_{e} \frac{T_{a}}{T_{h}} .
\end{aligned}
$$

The output voltage of the cell is then given by

$$
V=\frac{1}{e}\left(\varepsilon_{F}^{+}-\varepsilon_{F}^{-}\right)=\frac{1}{e}\left(1-\frac{T_{a}}{T_{h}}\right)\left(\hat{\varepsilon}_{e}-\hat{\varepsilon}_{h}\right) .
$$

It is important to stress why monoenergetic contacts allow for reversibly converting electrons from the hot temperature $T_{h}$ to the contact temperature $T_{a}$. In this respect, notice that (13) and (14) can be satisfied only for a single energy value. This mechanism is called reversible cooling at the contacts.

As stated in Section I, we emphasize that, in this cell, $\mathrm{eV}=$ $\varepsilon_{F}^{+}-\varepsilon_{F}^{-}$is not equal to the difference of the electrons and hole electrochemical potentials in the core material, which is zero. Elaborating on this model, it is possible to demonstrate that the efficiency limit for solar energy conversion for this device is $86.5 \%$ (assuming that the sun is at $6000 \mathrm{~K}$ and the cell at $300 \mathrm{~K}$ ) and is obtained for a bandgap $E_{G}=0$ [6], [15]. This 
model for the HCSC is known as the "impact-ionization model," and it can be shown that the current-voltage characteristics of the cell from which this efficiency is obtained are formulated from energy conservation principles with the only additional condition being the reversible cooling at the energy contacts previously mentioned [6], [15].

However, in the original paper in which Ross and Nozik [1] introduced HCSCs, the authors assumed that nonradiative generation-recombination mechanisms linking the $\mathrm{CB}$ and the VB did not exist. These mechanisms include, in particular, Auger recombination and impact ionization [the mechanisms illustrated in Fig. 2(c) and (d)]. We propose here-for the first time, to our knowledge- that in order for the following arguments to hold, it is also necessary to assume that Auger thermalization [the mechanism illustrated in Fig. 2(e)], which is also a mechanism linking electrons in the $\mathrm{CB}$ to electrons in the VB, does not exist either. Hence, for generality, instead of "nonradiative recombination processes," we should assume that "interband processes" do not exist or occur at a much slower rate than intraband processes. This is perhaps what one would naturally expect to be the case in conventional semiconductors, but, as explained in the following paragraphs, this leads to some difficulties within the HCSC model.

Following the same arguments that led to (4), the absence of interband processes implies that the electrochemical potential of the electrons in the $\mathrm{CB}, \mu_{i, C}$, and the electrochemical potential of the electrons in the VB, $\mu_{i, V}$, are related to the energy of the states by

$$
\begin{aligned}
& \mu_{i, C}=a_{C} \varepsilon_{i}+b_{C} \\
& \mu_{i, V}=a_{V} \varepsilon_{i}+b_{V}
\end{aligned}
$$

which implies that electrons in the CB can be regarded as having an energy-independent electrochemical potential and hot-carrier temperature given, respectively, by

$$
\begin{aligned}
& \mu_{e, C}=b_{C} /\left(1-a_{C}\right) \\
& T_{h, C}=T_{c} /\left(1-a_{C}\right) .
\end{aligned}
$$

Similarly, electrons in the VB can be described by an energyindependent electrochemical potential and hot-carrier temperature given by

$$
\begin{aligned}
& \mu_{e, V}=b_{V} /\left(1-a_{V}\right) \\
& T_{h, V}=T_{c} /\left(1-a_{V}\right) .
\end{aligned}
$$

This means that electrons and holes have, in principle, not only different electrochemical potentials $\left(\mu_{e, C}\right.$ and $\left.\mu_{e, V}\right)$ but also different hot-carrier temperatures $\left(T_{h, C}\right.$ and $\left.T_{h, V}\right)$. We emphasize that this reasoning is based on the fact that electrons in the $\mathrm{CB}$ are isolated from the electrons in the VB due to the absence of interband scattering processes; therefore, each set gets its own constants "a" (namely, $a_{C}$ and $a_{V}$ ) and "b" (namely, $b_{C}$ and $b_{V}$ ) that are not necessarily equal. However, as explained in the discussion that motivated (8), to have a solar cell, electrons must interact with photons and this interaction connects the $\mathrm{VB}$ with the $\mathrm{CB}$; therefore, electrons in the $\mathrm{VB}$ and the $\mathrm{CB}$ have a temperature equal to that of the photons. We can simply designate this common temperature as $T_{h}$, which implies that $a_{C}=a_{V}$. However, because electrons in the CB and VB now have different electrochemical potentials (because, in general, $b_{C} \neq b_{V}$ ), from (8), we now obtain

$$
\mu_{h v}=\mu_{e, C}-\mu_{e, V}
$$

which is not equal to zero. Therefore, under this model, called the "particle-conservation model," photons have a chemical potential that equals the electrochemical potential difference between electrons and holes. This difference is not equal to $\mathrm{eV}$ because the energy-selective contacts are still necessary to reversibly cool the electrons from their hot temperature to room temperature. The current-voltage characteristics of the cell cannot be obtained solely from energy-conservation rules; therefore, another equation is needed to calculate $\mu_{h v}$. This new equation (not included here), the one that gives the model its name, states that the difference between absorbed and emitted photons must equal the number of electrons flowing through the external circuit. This model is explained in detail in [7] by Würfel, who found peculiar results, including that the hot-carrier temperature of the emitted photons exceeds the sun's temperature at some points along the current-voltage characteristic of the cell as well as situations in which a complete current-voltage curve cannot be obtained. Given these unrealistic results, it is preferable to drive the HCSC toward operating conditions in which the impact-ionization mechanism applies. These conditions would be those in which intraband scattering processes are slowed to the point that their rate is comparable with that of the interband processes. By contrast, having $\mu_{e, C}-\mu_{e, V} \neq 0$ in the particle conservation model introduces an extra degree of freedom that results in an efficiency limit for the HCSC that is slightly higher using this model than using the impact-ionization model under maximally concentrated light.

Until now, electron interactions with phonons have been neglected. As discussed at the end of Section II, allowing the electrons to interact with phonons leads to $a_{C}=0$ and $a_{V}=0$. If this interaction is allowed, the HCSC concept is not possible because, according to (11), the electrons are not hot anymore and $T_{h}=T_{C}$. There are two methods to avoid this. One, obviously, is to assume the existence of a material in which electronphonon interaction has been suppressed or made much slower than electron-electron scattering. However, it must be remembered that we also wanted electron-electron interaction to be slowed in order to create the HCSC impact-ionization model. Thus, if we decrease the electron-electron interaction rate, it is likely that electron-electron interaction will conflict at some point with electron-phonon interaction because not all interactions can remain slow simultaneously.

The second method is to assume that electrons interact, not with all types of phonons but only with optical phonons [16]. Optical phonons are approximately stationary modes because their energy does not depend on the wave vector; thus, their group velocity in an ideal situation is zero. Therefore, they do not transport heat to the cell walls (this condition should ideally hold for all optical phonon branches ). By contrast, the dispersion curve of acoustic phonons is not flat; thus, they can transport heat toward the cell walls. Therefore, an acoustic phonon would be 
in thermal contact with these walls, in contact with the ambient or with heat sinks, and would be at room temperature. Because optical phonons would be isolated from acoustic phonons, optical phonons could be set at a higher temperature. In fact, from (10), they can be at the electron temperature $T_{h}$ while remaining consistent with phonons having a chemical potential of zero. Interaction of optical phonons with acoustic phonons would be suppressed by avoiding mechanisms such as Klemen's decay by the creation of phononic bandgaps. Allowing electrons to interact directly with acoustic phonons at room temperature would collapse the HCSC model.

\section{IMPACT-IONIZATION SOLAR CELl}

In the HCSC particle conservation model, we assumed that the impact-ionization rate (in fact, all interband processes) would be much slower than electron-electron interaction within bands (intraband processes). Instead of "energy-selective" contacts, we now assume that the cell is contacted with contacts selective to electrons and holes instead, i.e., with contacts that allow only electrons or holes to pass through. The resulting solar cell model will be the IISC or MEGSC. The practical difference between the two is that the IISC model is applied to bulk semiconductors [17] and MEGSC is applied to quantum dots [4], but fundamentally they can be considered the same model.

Electron- and hole-selective contacts have a wide range of energies in which electrons can pass freely from the semiconductor to the contact and vice versa. Consequently, electrons at the semiconductor CB must have the same Fermi-Dirac distribution function as the electron-selective contact and electrons at the VB must have the same Fermi-Dirac distribution function as the hole-selective contact. In particular, they are all at the contact temperature $T_{a}\left(a_{C}=a_{V}=0\right)$. However, the electrochemical potential of the VB and its contact may be different from that of the $\mathrm{CB}$ and its contact. By setting $a_{C}=a_{V}=0$ in (18) and (20) and using (17) and (19), we obtain in fact

$$
\begin{aligned}
& \mu_{e, C}=b_{C} \\
& \mu_{e, V}=b_{V} .
\end{aligned}
$$

In this cell, however, the electron-phonon interaction is not suppressed and this gives an additional reason to consider $a_{C}=$ $a_{V}=0$.

The voltage between terminals of this cell is $e V=\mu_{e, C}-$ $\mu_{e, V}$, but $\mu_{h v} \neq \mu_{e, C}-\mu_{e, V}$ because (8) does not govern the photon interaction. Instead, it is governed by

$$
e_{1}+e_{2}+\cdots+e_{M}+h v(\varepsilon) \leftrightarrow e_{M+1}+e_{M+2}+\cdots+e_{2 M}
$$

where $M$ is the number of electron-hole pairs that a photon with energy $\varepsilon$ is capable of generating. This interaction between photon and electrons is shown in Fig. 4(a). Thus, we obtain

$$
\mu_{h v}=M\left(\mu_{e, C}-\mu_{e, V}\right) .
$$

This result was obtained in [3] and [18] using other thermodynamic arguments. Obtaining the result in (25) from (24) provides new insight because (24) is conceptually different from

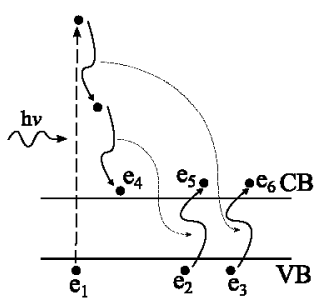

(a)

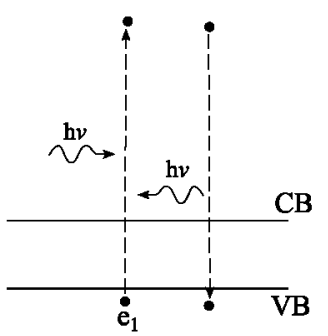

(b)
Fig. 4. (a) Illustration of the mechanism leading to the generation of multiple electron-hole pairs after the absorption of a single photon. (b) Direct radiative recombination of a high-energy electron-hole pair.

(8). The difference is that in (8), one high-energy photon can generate a high-energy electron-hole pair and this electron-hole pair can then recombine to generate a high-energy photon [see Fig. 4(b)], whereas in (24), one high-energy photon can generate one high-energy electron-hole pair, but this electron-hole pair cannot recombine to generate a high-energy photon unless the electron-hole pair has been created by a multiple-impactionization process. Equation (24) and Fig. 4(a) would then represent a multiple-particle collision process and not a succession of events in time, and would become more and more unlikely as the number of particles involved increases. Thus, in the same way that nonradiative recombination is a loss mechanism for cells working at the radiative limit, radiative recombination of high-energy electron-hole pairs becomes a loss mechanism for cells based on impact-ionization or multiple-exciton generation. Equation (25) also leads to a possible means of experimental verification of the operation of an IISC/MEGSC because the luminescent emission of these high-energy photons, well above the bandgap and following the relationship in (25), should be detectable experimentally. An additional argument for considering the phenomena represented in Fig. 4(a) as a multiple-particle collision process is that, otherwise, we should accept interband processes as the ones in Fig. 2(d) and (c) as occurring in the same time scale what would take us, following the arguments that lead us to (7), to the impossibility for having different electron and hole electrochemical potentials and, therefore, to a solar cell with an output voltage different from zero.

\section{Conventional Single-Gap Solar Cell}

The single-gap solar cell model, whose efficiency at the radiative limit was first studied by Shockley and Queisser (S\&Q) [5], can now be obtained from the IISC/MEGSC model by setting $M=1$. In a conventional single-gap solar cell, electrons and holes have cooled to room temperature after conventional contacts selective to electrons and holes have been placed. Electrons also cool down if the electron-phonon interaction is allowed [see (10)]. As in an IISC/MEGSC, electrons in the CB and VB obtain their own electrochemical potential. The $S \& Q$ model is perhaps the one most widely used to study the efficiency limit of solar cells, and we do not elaborate on it here. To calculate this limit, nonradiative recombination is assumed to not exist. One of the authors of this paper reviewed it extensively in [19]. The S\&Q model is also the building block used to study the efficiency 


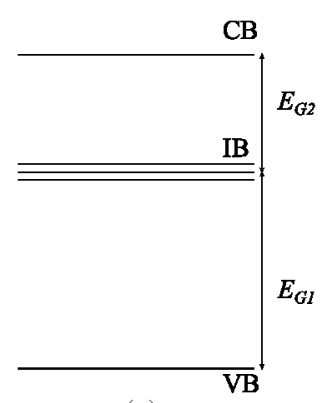

(a)

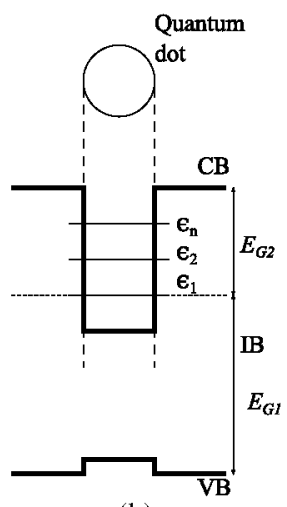

(b)
Fig. 5. (a) Energy bandgap diagram of an IBSC. (b) Simplified energy-band structure arising from the implementation of the IBSC concept with a quantum dot where several energy levels emerge.

limit of multijunction solar cells [20], [21]. Traditionally, the IISC/MEGSC and HCSC models were obtained from the singlegap solar cell model by following a bottom-up approach. Here, on the other hand, we have followed a top-down approach in which the single-gap solar cell model has been obtained starting from a general core photovoltaic material.

\section{INTERMEDiATE-BAND SOLAR CELL}

The IBSC model [2] can now be considered a generalization of the single-gap solar cell model by assuming the existence of a material with two bandgaps, $E_{G 1}$ and $E_{G 2}$, instead of one [see Fig. 5(a)]. Energy levels are now grouped into the CB, $\mathrm{VB}$, and IB. Electron-phonon interaction within each band is allowed so that electrons are at room temperature. In an ideal cell, interband processes are assumed to be much slower than intraband processes so that each band acquires its own electrochemical potential. As with a single-gap solar cell, this hypothesis is equivalent to neglecting nonradiative recombination. In the particular case where the IBSC model is implemented with quantum dots [22] [see Fig. 5(b)], our arguments in previous sections also indicate the plausibility that each energy level in the QD, $\varepsilon_{1}, \varepsilon_{2}, \ldots, \varepsilon_{n}$, acquires its own electrochemical potential [23] if these levels are spaced far enough apart in terms of energy to prevent electron-phonon interactions from causing electrons to transit from one state to another. To make an actual cell, selective contacts are made to electrons in the CB and VB, while the IB is left noncontacted. This can be achieved by simply sandwiching the IB material between conventional p- and n-type semiconductors [9]. Noncontacting the IB is an additional argument for allowing energy levels in the QD having their own electrochemical potential.

\section{SUMMARY AND CONCLUSION}

We have revisited how electrochemical potentials for electrons and photons are distributed in the HCSC, IISC/MEGSC, single-gap solar cell, and IBSC models. We started our discussion with a core material in which only electron-electron interactions existed and arrived at the other cell models by

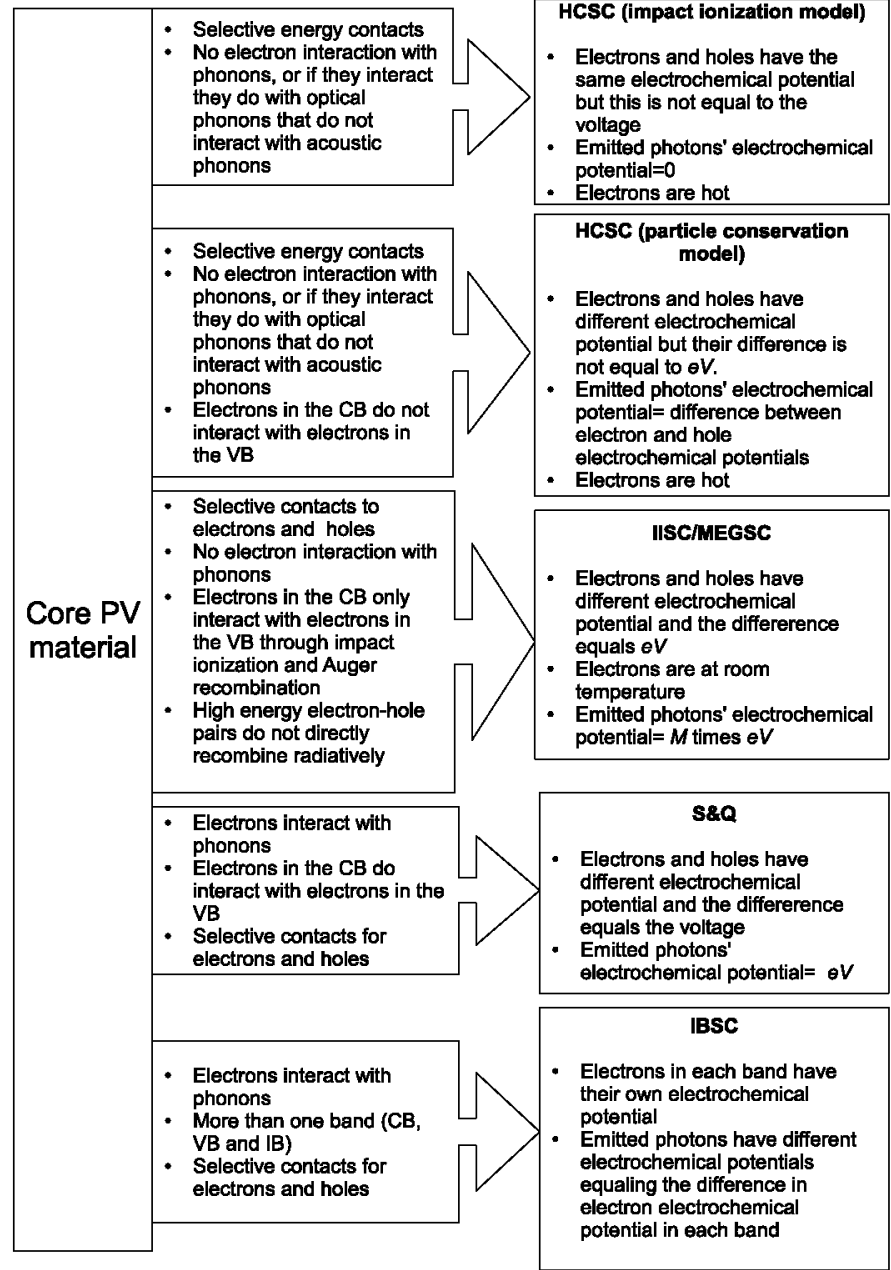

Fig. 6. Summary of the novel cells discussed in this study and how electron, hole, and photon electrochemical potentials are distributed: IBSC, S\&Q model, IISC/MEGSC, and HCSC.

imposing different restrictions on this electron-electron interaction, assuming energy-selective contacts or electron-selective contacts and turning $\mathrm{ON}$ and OFF different electron-phonon and electron-photon interactions. Throughout this paper, we have explained the mechanisms by which electrons in each band can be described by a single electrochemical potential, whether electrons can be considered hot and whether the photon electrochemical potential equals $e V$. The discussion presented in this paper is summarized in the form of a tree in Fig. 6. The trunk is the "core photovoltaic material" and the branches are various novel solar cells that stem from the hypotheses made.

When selective "energy" contacts are attached to the core material and electron-acoustic phonon interaction is neglected, we obtain the HCSC. Still, there are two models for this cell: the impact-ionization model [6] and the particle conservation model [7]. In the first, electrons and holes share the same electrochemical potential; in the second, they do not. The chemical potential of the photons emitted by the cell is equal to the electron and hole electrochemical potential difference; therefore, this chemical potential is equal to zero in the impact-ionization model and, in general, different from zero according to the 
particle conservation model. In both cases, and unlike the usual single-gap solar cell, the electron and hole electrochemical potential difference in the core material is not equal to $\mathrm{eV}$.

When we make selective contacts for the flow of electrons and holes (instead of selective "energy" contacts), we arrive at IISC/MEGSC. In this case, electrons and holes have different quasi-Fermi levels, and their split in the core material is now equal to $\mathrm{eV}$. The chemical potential of the emitted photons is $M$ times $e V$, where $M$ is the carrier multiplication factor (the number of electron-hole pairs generated per absorbed photon).

When selective contacts to electrons and holes are made and the electron-phonon interaction is not neglected, we arrive at the conventional single-gap solar cell model [5]. Electrons and holes have different electrochemical potentials, and their difference in the core material equals $e V$. The chemical potential of the emitted photons also equals $e V$. When several single-gap solar cells are grouped, they form a multijunction solar cell. When we add a third band (the IB) instead of just the CB and VB, we obtain the IBSC in which electrons in each band have their own electrochemical potentials.

In reviewing these topics, we have found some subtleties that, to our knowledge, were previously disregarded. Thus, we have found that Auger thermalization processes must be minimized to implement an HCSC based on the particle-conservation model. We have also found that in an ISC/MEGSC cell, radiative recombination of the high-energy electron-hole pairs (or excitons) in one step [see Fig. 4(b)] is a loss mechanism for this cell and suggest that the absorption of a high-energy photon and creation of multiple electron-hole pairs in this cell should be considered a multiple-particle process rather than a succession of steps in order to explain why the photon chemical potential in this cell equals $M$ times $e V$.

\section{REFERENCES}

[1] R. T. Ross and A. J. Nozik, "Efficiency of hot-carrier solar energy converters," J. Appl. Phys., vol. 53, pp. 3813-3818, 1982.

[2] A. Luque and A. Martí, "Increasing the efficiency of ideal solar cells by photon induced transitions at intermediate levels," Phys. Rev. Lett., vol. 78, pp. 5014-5017, 1997.

[3] J. H. Werner, R. Brendel, and H. J. Queisser, "Radiative efficiency limit of terrestrial solar-cells with internal carrier multiplication," Appl. Phys. Lett, vol. 67, pp. 1028-1030, Aug. 1995.

[4] A. J. Nozik, "Quantum dot solar cells," Physica E, vol. 14, pp. 115-120, 2002.

[5] W. Shockley and H. J. Queisser, "Detailed balance limit of efficiency of p-n junction solar cells," J. Appl. Phys., vol. 32, pp. 510-519, 1961.
[6] P. Würfel, "Solar energy conversion with hot electrons from impact ionisation," Solar Energy Mater. Solar Cells, vol. 46, pp. 43-52, Apr. 1997.

[7] P. Würfel, A. S. Brown, T. E. Humphrey, and M. A. Green, "Particle conservation in the hot-carrier solar cell," Progr. Photovoltaics: Res. Appl, vol. 13, pp. 277-285, 2005.

[8] G. J. Conibeer, D. König, M. A. Green, and J. F. Guillemoles, "Slowing of carrier cooling in hot carrier solar cells," Thin Solid Films, vol. 516, pp. 6948-6953, 2008.

[9] A. Luque and A. Martí, "A metallic intermediate band high efficiency solar cell," Progr. Photovoltaics: Res. Appl., vol. 9, pp. 73-86, 2001.

[10] R. Strandberg and T. W. Reenaas, "Limiting efficiency of intermediate band solar cells with spectrally selective reflectors," Appl. Phys. Lett., vol. 97, pp. 031910-1-031910-3, 2010.

[11] R. J. Ellingson, M. C. Beard, J. C. Johnson, P. R. Yu, O. I. Micic, A. J. Nozik, A. Shabaev, and A. L. Efros, "Highly efficient multiple exciton generation in colloidal $\mathrm{PbSe}$ and $\mathrm{PbS}$ quantum dots," Nano Lett., vol. 5, pp. 865-871, May 2005.

[12] O. E. Semonin, J. M. Luther, S. Choi, H.-Y. Chen, J. Gao, A. J. Nozik, and M. C. Beard, "Peak external photocurrent quantum efficiency exceeding $100 \%$ via MEG in a quantum dot solar cell," Science, vol. 334, pp. 15301533, Dec. 16, 2011.

[13] D. Kondepudi and I. Prigogine, Modern Thermodynamics. Chichester, U.K.: Wiley, 1998, p. 87.

[14] M. 1. Bellac, F. Mortessagne, and G. G. Batrouni, Equilibrium and Non-Equilibrium Thermodynamics. Cambridge, U.K.: Cambridge Univ. Press, 2004, p. 336.

[15] A. Luque and A. Martí, "Theoretical limits of photovoltaic energy conversion and new-generation solar cells," in Handbook of Photovoltaic Science and Engineering, A. Luque and S. Hegedus, Eds. Chichester, U.K.: Wiley, 2011.

[16] G. J. Conibeer, J. F. Guillemoles, and M. A. Green, "Phononic band gap engineering for hot carrier solar cell absorbers," in Proc. 20th Eur. Photovoltaic Solar Energy Conf, 2005, pp. 35-38.

[17] R. Brendel, J. H. Werner, and H. J. Queisser, "Thermodynamic efficiency limits for semiconductor solar cells with carrier multiplication," Solar Energy Mater. Solar Cells, vol. 41-42, pp. 419-425, Jun. 1996.

[18] A. Luque and A. Martí, "Entropy production in photovoltaic conversion," Phys. Rev. B, vol. 55, pp. 6994-6999, 1997.

[19] G. L. Araujo and A. Marti, "Absolute limiting efficiencies for photovoltaic energy conversion," Solar Energy Mater. Solar Cells, vol. 33, pp. 213-240, 1994.

[20] A. Devos and H. Pauwels, "On the thermodynamic limit of photo-voltaic energy-conversion," Appl. Phys., vol. 25, pp. 119-125, 1981.

[21] A. Martí and G. L. Araújo, "Limiting efficiencies for photovoltaic energy conversion in multigap systems," Solar Energy Mater. Solar cells, vol. 43, pp. 203-222, 1996

[22] A. Martí, L. Cuadra, and A. Luque, "Quantum dot intermediate band solar cell," in Proc. 28th IEEE Photovoltaic Spec. Conf., 2000, pp. 940-943.

[23] A. Luque, P. G. Linares, E. Antolín, E. Cánovas, C. D. Farmer, C. R. Stanley, and A. Martí, "Multiple levels in intermediate band solar cells," Appl. Phys. Lett., vol. 96, pp. 013501-1-013501-3, 2010. 